INPLASY

PROTOCOL

To cite: Tang et al.

Acupuncture for drooling in children with cerebral palsy: a systematic review protocol. Inplasy protocol 2020110024. doi:

10.37766/inplasy2020.11.0024

Received: 06 November 2020

Published: 06 November 2020

Corresponding author: Jun Xiong

xiongjun196071@163.com

Author Affiliation: Jiangxi University of Traditional Chinese Medicine

Support: 1050Project: 5141900101.

Review Stage at time of this submission: The review has not yet started.

Conflicts of interest: The authors declare no conflicts of interest.

\section{Acupuncture for drooling in children with cerebral palsy: a systematic review protocol}

Tang, $\mathrm{GH}^{1} ;$ Xiong, $\mathrm{J}^{2}$.

Review question / Objective: This review in an effort to summarize the available evidence from randomized controlled trial for the efficacy of acupuncture therapy for children with cerebral palsy salivation.

Condition being studied: Cerebral palsy is a syndrome caused by non-progressive brain damage in a developing fetus or baby, the main symptoms were central dyskinesia and abnormal postures, children with dysphagia and dysphagia in oral stage cannot clear saliva through swallowing, leading to salivation of varying degrees. Western medicine and psychotherapy are not universally effective in treating this disease, Acupuncture reported to be efficacious and widely used for the treatment of children with cerebral palsy salivation. This review aims to summarize the available evidence from randomized controlled trial for the efficacy of acupuncture therapy for children with cerebral palsy salivation.

INPLASY registration number: This protocol was registered with the International Platform of Registered Systematic Review and Meta-Analysis Protocols (INPLASY) on 06 November 2020 and was last updated on 18 November 2020 (registration number INPLASY2020110024).

\section{INTRODUCTION}

Review question / Objective: This review in an effort to summarize the available evidence from randomized controlled trial for the efficacy of acupuncture therapy for children with cerebral palsy salivation.

Condition being studied: Cerebral palsy is a syndrome caused by non-progressive brain damage in a developing fetus or baby, the 
main symptoms were central dyskinesia and abnormal postures, children with dysphagia and dysphagia in oral stage cannot clear saliva through swallowing, leading to salivation of varying degrees. Western medicine and psychotherapy are not universally effective in treating this disease, Acupuncture reported to be efficacious and widely used for the treatment of children with cerebral palsy salivation. This review aims to summarize the available evidence from randomized controlled trial for the efficacy of acupuncture therapy for children with cerebral palsy salivation.

\section{METHODS}

Participant or population: Children with cerebral palsy salivation.

Intervention: Needle acupuncture, body acupuncture, electro-acupuncture, auricular acupuncture, acupressure, point injection, or any combination of the above.

Comparator: Western medicine, placebo, sham acupuncture, rehabilitation training, no treatment, or any combination of these.

Study designs to be included: All randomized controlled trial on the use of acupuncture for children with cerebral palsy salivation.

Eligibility criteria: Published systematic reviews which were reported in Chinese or English, and meet the "PICOS", will be considered for inclusion in this review.

Information sources: Pubmed, Embase, Cochrane Library, Chinese Biomedical Literatures Database(CBM), China National Knowledge Infrastructure ( CNKI), Wang Fang Database ( WF), Chinese Scientific Journal Database (VIP).

Main outcome(s): The effective rate.

Quality assessment / Risk of bias analysis: Assessment of Multiple Systematic Reviews 2 (AMSTAR-2) measurement tool, Preferred Reporting Items for Systematic
Reviews and Meta-Analyses (PRISMA), Grading of Recommendations Assessment, Development and Evaluation(GRADE) approach.

Strategy of data synthesis: Strategy of data synthesis: Meta analysis was performed using RevMan 5.4 provided by the Cochrane collaboration network. Relative risk (RR) was used for the two categorical variables, and mean difference (MD) was used for the Continuous variables. Both were expressed with $95 \%$ confidence intervals $(\mathrm{Cl})$. The heterogeneity test between the results of the included studies was performed using the 12 test. The 12 value reflects the proportion of the total variation in the effect size due to the existence of heterogeneity. (12> 50\%, indicating that heterogeneity is more obvious. If there is not obvious heterogeneity between the research results (12 50\%), the source of the heterogeneity is analyzed first, which may lead to heterogeneity factors for subgroup analysis. If statistical heterogeneity exists in each subgroup without clinical heterogeneity, a random effects model is used for analysis. If the heterogeneity is too large and the results cannot be combined, a descriptive analysis is used and a sensitivity analysis is performed if necessary.

Subgroup analysis: Subgroup analysis will be handled according to the differences in acupuncture, patient conditions, and control. If the necessary data are available, subgroup analysis will be carried out according to different factors as follows: 1 . Control interventions (eg, Needle acupuncture, electro- acupuncture, auricular acupuncture, acupressure, point injection, or any combination of the above.). 2. Type of acupuncture (eg, needle acupuncture, electro-acupuncture, auricular acupuncture).

Sensibility analysis: To assess the influence of each individual study, leave-one-out sensitivity analysis was performed iteratively by removing one study at a time to confirm that the findings were not influenced by any single study. 
Country(ies) involved: China.

Keywords: acupuncture; children; cerebral palsy salivation; systematic review.

Contributions of each author:

Author 1 - Genhua Tang - the author drafted the manuscript.

Email: tanggenhua1015@163.com

Author 2 - Jun Xiong - revise this protocol.

Email: xiongjun196071@163.com 